\title{
Trauma Team Activation for Geriatric Trauma at a Level II Trauma Center: are the Elderly Under-triaged? \\ Grant Buchanan, MD $^{1}$, Daniel Kahn, MSIV ${ }^{1}$, Harry Burke, MSII ${ }^{1}$, Brian Czarkowski, MD ${ }^{1}$, Richard Boe, MD ${ }^{1}$, Milad Modarresi, MD ${ }^{1}$, Franklin D. Shuler, MD, $\mathrm{PhD}^{1}$
}

\section{Author Affiliations:}

1. Marshall University Joan C. Edwards School of Medicine, Huntington, West Virginia

Dr. Shuler serves on the editorial committee of the Marshall Journal of Medicine. Other authors have no conflicts of interest to disclose.

\section{Corresponding Author:}

Milad Modarresi, MD

Department of Orthopaedic Surgery

Marshall University Joan C. Edwards School of Medicine

Huntington, West Virginia

Email: $\underline{\text { modarresi@marshall.edu }}$ 


\section{Abstract}

Geriatric patients often sustain life-threatening injuries from minor trauma. A growing body of research suggests that these patients are often under-triaged in the emergency setting. The purpose of this research was to evaluate whether or not geriatric trauma patients are undertriaged at a community based level II trauma center.

1434 trauma patients over the age of 65 presenting from 2010-2015 were retrospectively reviewed from the Cabell Huntington Hospital trauma registry and analyzed for age, gender, arrival type, ED response, Glasgow Coma Scale (GCS), Injury Severity Score (ISS), injury cause, ICD-9 diagnosis codes, and mortality. Under-triage and over-triage rates were determined using the Cribari method (under-triage $=$ ISS $\geq 16$ without full trauma team activation [TTA]; Over-triage $=$ ISS $\leq 15$ with full TTA).

The under-triage rate was $9.5 \%$ (132/1393) with the majority of under-triaged patients having head trauma $(\mathrm{n}=423)$. There were 371 head trauma patients with a recorded GCS and analysis shows those with a GCS $\geq 13$ had a $1.2 \%$ mortality risk $(n=326$; ISS 10.2$)$, but that risk drastically increased to $60 \%$ with GCS $\leq 12$ (n=45; ISS 21.5$)$. Of the 45 patients with GCS $\leq 12$, only $4 \%$ had priority 1 TTA using the current protocol $(2 / 45)$.

The American College of Surgeons-Committee of Trauma (ACS-COT) recommends an acceptable under-triage rate of $<5 \%$. In order to improve geriatric care and reduce under-triage rates, we recommend that an age-based criteria be added to our TTA protocol at our community based level II trauma center: priority 1 TTA for all patients 65 years or older sustaining head trauma with a GCS $\leq 12$ or suspicion of intracranial hemorrhage.

\section{Keywords}

Geriatric, trauma, trauma team activation, head trauma, intracranial hemorrhage, GCS, ISS

\section{Introduction}

West Virginia has the second oldest population in the country based on percentage of residents $\geq$ 65 years of age. ${ }^{1}$ This elderly demographic dramatically impacts delivery of trauma care as geriatric trauma patients can sustain life-threatening injuries from even minor trauma. ${ }^{2,3}$ Patients who do not undergo a trauma team activation (TTA), despite sustaining injuries consistent with TTA, are considered to be under-triaged and a growing body of research indicates geriatric patients are often under-triaged in an emergency setting. ${ }^{4-13}$ Unfortunately, the elderly have no specific and standard criteria for TTA despite having higher hospitalization and mortality rates. ${ }^{4-}$ 13 As a result, it is less likely for them to undergo TTA. ${ }^{3,10,14,15}$ Several studies have even recommended advanced age to be made an additional criterion for TTA with the hope to decrease under-triaging and improve the emergent care of geriatric patients. ${ }^{4,5,8,9,11}$

This study evaluates the TTA response to geriatric patients presenting to a community based level II trauma center (Cabell Huntington Hospital [CHH]) over the past 5 years (2010-2015). Two critical factors in data analysis are the use of the gold standards for evaluating neurological 
and physical trauma in the emergency setting: The Glasgow Coma Scale (GCS) and the Injury Severity Score (ISS), respectively. The GCS focuses on a three-tiered neurological summary based upon ocular, verbal, and motor elements. This results in Mild (14-15), Moderate (9-13), or Severe (0-8) injury status. ${ }^{16}$ GCS directly correlates to disability and mortality outcomes and functions as an index of injury severity. ${ }^{16}$ The ISS is a more complex rating system that uses Abbreviated Injury Scales (AIS) for six body regions of: head and neck, face, chest, abdomen or pelvis, extremities or pelvic girdle, and external surface in order to create generalized injury score. ${ }^{17,18}$ ISS is derived from sum of the three highest AIS scored body regions that are squared individually. An ISS score of 15 or higher is considered to be major trauma and typically involves TTA.

The purpose of this research was to evaluate whether or not geriatric trauma patients are undertriaged at a community based level II trauma center. It is our hypothesis that geriatric patients are under-triaged at $\mathrm{CHH}$ and an age specific TTA criterion is warranted.

\section{Methods}

This research study received Institutional Review Board approval (702627). Data and statistics were retrospectively reviewed and calculated from CHH trauma registry for January 2010 through December 2014. Other data analysis includes: gender, arrival type, ED response, GCS, ISS, cause of injury, ICD-9 diagnosis codes, and mortality.

Under-triage and over-triage rates were determined using the Cribari method: under-triaged patients are defined as ISS $\geq 16$ without full or partial TTA; over-triaged patients are defined as ISS $\leq 15$ with full TTA. CHH procedures are set in accordance with the American College of Surgeons (ACS-COT) at $<5 \%$ for under-triage and $<50 \%$ for over-triage. The ACS-COT criteria for priority 1 (full) TTA is shown:

\section{Criteria for priority 1 (full) Trauma Team Activation}

- Confirmed blood pressure less than $90 \mathrm{mmHg}$ at any time in adults and age specific hypotension in children

- Gunshot wounds to the neck, chest, or abdomen or extremities proximal to the elbow/knee

- Glasgow Coma Scale score less than 9 with mechanism attributed to trauma

- Transfer patients from other hospitals receiving blood to maintain vital signs

- Intubated patients transferred from the scene, - OR -

- Patients who have respiratory compromise or are in need of an emergent airway -Includes intubated patients who are transferred from another facility with ongoing respiratory compromise (does not include patients intubated at another facility who are now stable from a respiratory standpoint)

- Emergency physician's discretion ${ }^{19}$ 


\section{The criteria for priority 2 (partial) TTA}

- GCS $\geq 8$ and $\leq 13$

- Respiratory rate $<10$ or $>29$ per minute in adults and children $>5$ years of age.

- Anatomy of Injury:

○ Flail chest

- 2 or more obvious proximal long bone fractures

- Crush, degloved, or mangled extremity

- Amputation proximal to the wrist or ankle

- Penetrating trauma to head, neck, torso, groin, or extremity proximal to the elbow or knee

- Drowning/near drowning (if do not meet P1 criteria)

- Hanging by the neck

- Pelvic fracture (except those sustained from same level falls)

- Open or depressed skull fracture

○ Paralysis

- Trauma with combination of burns $>20 \%$ or involving face or airway or children $<5$ years of age with $>10 \%$ BSA burns

$\circ$ Time sensitive extremity injury

- Mechanism

○ Falls

- Adults $>20$ feet ( 1 story $=10$ feet $)$

- Children $>10$ feet or 2-3 times the height of the child

- High risk auto crash

- Intrusion > 12 inches into occupant site; > 18 inches at any site

- Ejection (partial or complete) from automobile

- Death in same passenger compartment

○ Auto vs. pedestrian/bicyclist

- Thrown

- Run over

- Or significant (>20 mph) impact

○ Motorcycle crash over $20 \mathrm{mph}$

- ATV crash with separation of rider and vehicle

- Priority 2 TTA patients with single injuries or in combination with other pathophysiologic condition present significant risk to their lives or well-being and require the expert evaluation of the trauma surgeon. These patients are admitted directly to the trauma service until it can be determined that they can be adequately cared for by subspecialties. At that point, the trauma surgeon may transfer the patient to the appropriate clinical service.

*Please note that there is no age related activation criteria based on geriatric trauma patients.

Average ISS and mortality rates were calculated for the most common ICD-9 diagnoses and GCS values were collected. Statistical analysis was completed using a confidence interval of $95 \%$. 


\section{Results}

There were 1,434 trauma patients $\geq 65$ years of age admitted during the 5 year study period. Full TTA was seen in 41 patients $(2.9 \%)$. The remaining 1393 patients received partial, or no TTA $(97.1 \%)$. Characteristics of the entire study population are shown in Tables 1 and 2.

Table 1: Average ISS and Mortality by diagnosis. The greatest mortality rate was seen for geriatric trauma patients with ICH $(16.1 \%)$

\begin{tabular}{|l|l|l|l|l|}
\hline Diagnosis & $\mathbf{n}$ & Average ISS & $\mathbf{9 5 \%} \mathbf{C l}$ & \% Mortality \\
\hline Intracranial Hemorrhage & 149 & 17.4 & 1.3 & 16.1 \\
\hline Pneumo/hemothorax & 39 & 16.7 & 3.1 & 12.8 \\
\hline Rib fracture & 139 & 11.5 & 1.3 & 5.8 \\
\hline Head Trauma (explanation in text) & 423 & 11.2 & 0.8 & 7.8 \\
\hline Vertebral fracture & 178 & 10.0 & 1.1 & 3.9 \\
\hline Hip fracture & 409 & 9.6 & 0.3 & 3.2 \\
\hline Concussion & 200 & 7.4 & 0.8 & 2.5
\end{tabular}

Head trauma, as defined by ICD-9 (any form of intracranial hemorrhage, skull/facial fracture, head contusion, cerebral contusion, or concussion), was the most common diagnosis for entire study population ( $n=423 / 1434$; Table 1). Specifically, the diagnosis of intracranial hemorrhage had the highest average ISS, 17.4, and the highest mortality rate, 16.1\% ( $n=149$; Table 1$)$. Pneumo/hemothorax had the second highest average ISS of 16.7, including a mortality rate of $12.8 \%(n=39)$. GCS were evaluated in tandem with average ISS for geriatric trauma patients with head trauma. (Table 2)

Table 2: GCS effect on mortality in head injured patients. For 52 head trauma patients, GCS was not recorded in the medical record. The remaining 371 patients had an assessment of the effect of GCS on mortality risk.

\begin{tabular}{|l|l|l|l|l|}
\hline GCS & $\mathbf{n}$ & Average ISS & $\mathbf{9 5 \%} \mathbf{C l}$ & \% Mortality \\
\hline$\leq 12$ & 45 & 21.5 & 2.9 & 60.0 \\
\hline$\geq 13$ & 326 & 10.2 & 0.8 & 1.2 \\
\hline
\end{tabular}

The analysis revealed 45 patients with GCS of $\leq 12$ had an average ISS of 21.5 and mortality rate of $60.0 \%$. In addition, the 326 patients who sustained head trauma, but exhibited a GCS of $\geq 13$, had a lower average ISS of 10.2 , with a significantly lower mortality rate at $1.2 \%$. It is interesting to note that the current protocol for partial TTA is GCS $\geq 8$ and $\leq 13$. Our data demonstrates a much higher mortality risk in the patients with head injury with GCS $\leq 12$.

Further analysis of data, as shown in Table 3, discovered that in the subset of head injured patients with $60 \%$ mortality risk, only $4 \%(2 / 45)$ had priority 1 TTA.

Table 3: GCS $\leq 12$ and current TTA rates for head injured geriatric trauma patients.

\begin{tabular}{l|l|l|l|} 
GCS & n & \% Mortality & Priority 1 TTA \\
$\leq 12$ & 45 & 60.0 & 2
\end{tabular}


There were 41 full TTA in our 1434 study population; 1393 with partial or no TTA. The Cribari method is demonstrated in Table 4. Under-triage (ISS $\geq 16$ with partial or no TTA at a goal of $\leq$ $5 \%$ ) occurred $9.5 \%$ of the time (132/1393), while over-triage (ISS $\leq 15$ with full TTA at a goal of $\leq 50 \%) 36.6 \%$ of the time $(15 / 41)$. The under-triage rate of $9.5 \%$ is much higher than the recommended ACS standard.

Table 4: Cribari Method. Showing under and over-triage percentage.

\begin{tabular}{|l|l|l|l|l|}
\hline Cribari Method & definition & Calculation & $\%$ & ACS Standard \\
\hline Under-triage & $\begin{array}{l}\text { ISS } \geq 16 \text { without full } \\
\text { or partial TTA }\end{array}$ & $132 / 1393$ & $9.5^{*}$ & $\leq 5 \%$ \\
\hline Over-triage & ISS $\leq 15$ with full TTA & $15 / 41$ & 36.6 & $\leq 50 \%$
\end{tabular}

The top 10 under-triaged diagnoses are highlighted in Figure 1. Head trauma was the most common injury seen in under-triaged patients, followed by intracranial hemorrhage, and vertebral fracture. This figure further shows the higher number of geriatric patients with head trauma being under-triaged compared to other injuries. It must be noted that multiple diagnoses can be realized for each patient.

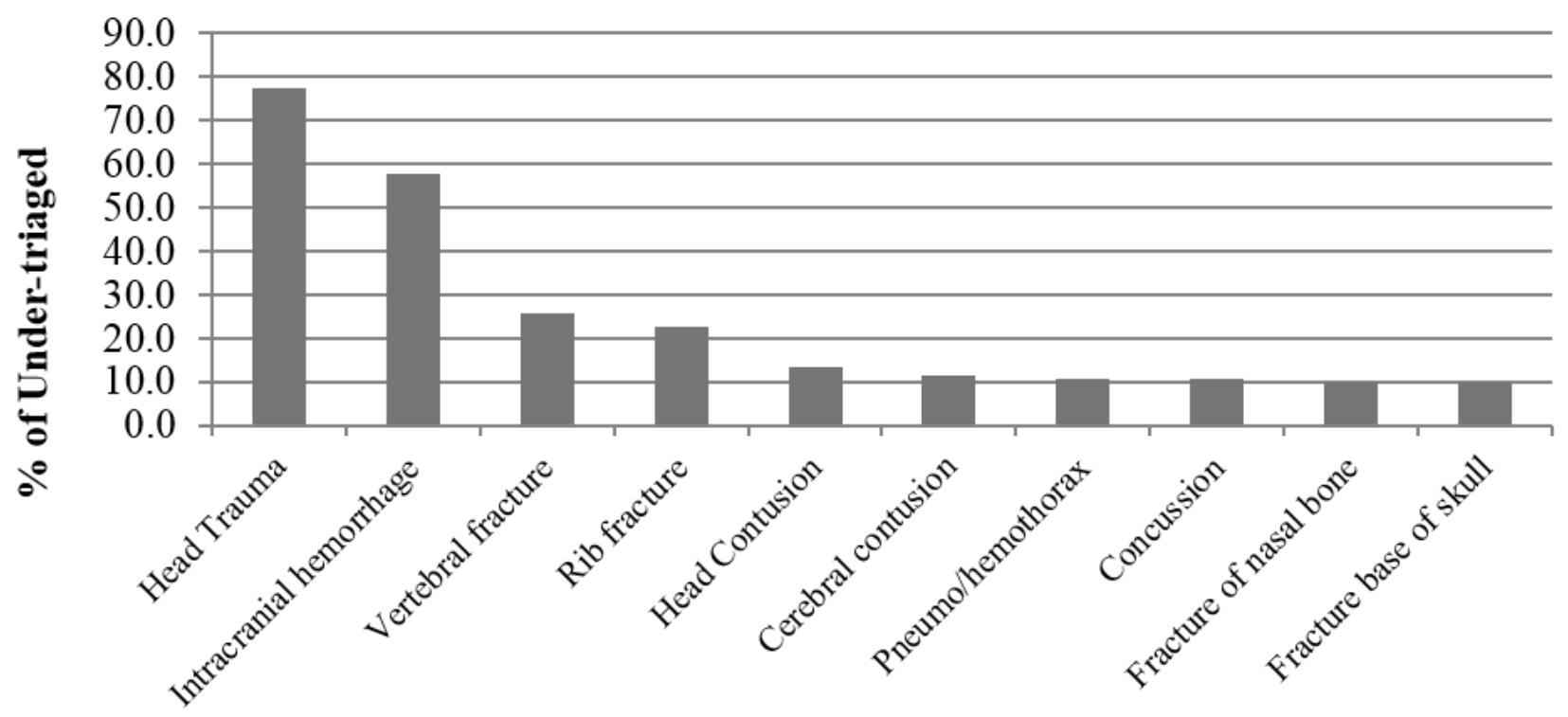

Figure 1: Top 10 diagnosis of under-triaged patients $(\mathrm{n}=132)$. Multiple diagnosis can be realized for each patient. Head trauma $(\mathrm{n}=102 ; 77 \%)$; ICH $(\mathrm{n}=63 ; 48 \%)$; Vertebral fracture $(n=34 ; 26 \%)$; Rib fracture $(n=30 ; 23 \%)$; Head contusion $(n=18 ; 14 \%)$; Cerebral contusion $(n=17$; $13 \%)$; Pneumo/hemothorax $(n=15 ; 11 \%)$; Concussion $(n=14 ; 11 \%)$; Nasal bone fracture $(n=14$; $11 \%)$ Base of skull fracture $(n=13 ; 10 \%)$

\section{Discussion}

Trauma is generally thought of as a "young person's disease", however, the expanding elderly population challenges this assertion. It is estimated that $19.3 \%$ of the total population will be $\geq$ 65 years of age by $2030 .^{3,20,21}$ Therefore, it is important to analyze TTA protocols to ensure adequate response for a population with greater co-morbidities and higher mortality rates. ${ }^{2-4,22-24}$ 
TTA, when initiated, is either a priority 1 (full) or priority 2 (partial) activation and ideally correlates with injury severity. It is concerning that current TTA criteria fails to consider the elderly's diminished physiologic response that ultimately leaves them more prone to suffer serious injury from even minor trauma. ${ }^{2,3}$ One has to consider frailty of the geriatric population as a major contributory factor when assessing TTA protocols. Clegg et al. describes frailty as "a state of increased vulnerability to poor resolution of homeostasis after a stressor event, which increases the risk of adverse outcomes, including falls, delirium, and disability. ${ }^{, 25,26}$ Frailty has a huge impact on geriatric patients as it disproportionately increases with age >65 and is often associated with polytraumatic events. ${ }^{6,25,26}$ Even with identical mean ISS values, geriatric patients suffer poorer outcomes when compared to younger demographic; as a person ages they have an increased vulnerability to injury and shock. ${ }^{3,4,22,27-29}$ Considering the paucity of data on under-triaged geriatric patients, it is vital to incorporate age-specific guidelines in TTA criteria in order to improve geriatric trauma outcomes. ${ }^{2,7,11,22,30}$

Using the Cribari method, our data analysis revealed the under-triage rate to be $9.5 \%$; significantly greater than the $<5 \%$ standard set by ACS. Head trauma was the most common diagnosis in under-triaged patients, while intracranial hemorrhage was associated with the highest ISS value and mortality rate (Table 2). This could be attributed to higher prevalence of antiplatelet/anticoagulation therapy in geriatrics. Both aspirin and warfarin are associated with increased mortality, but only aspirin has been associated with increased intracranial hemorrhage. ${ }^{27,31}$ Although intracranial hemorrhage results in higher mortality rates, traumatic brain injury (TBI) remains a critical prognostic factor. It was shown even mild TBIs are independent risk factors for increased mortality. ${ }^{32}$ When comparing age stratified TBI patients, geriatrics have nearly a $75 \%$ higher mortality rate than their younger counterparts. ${ }^{32-36}$ Further analysis demonstrates GCS as a precarious predictive factor in determining outcomes in the geriatric population. For example, a 60\% mortality rate was highlighted in Table $\mathbf{2}$ in those patients with head trauma and a GCS $\leq 12$ (ISS was 21.5; well above the major trauma value of 15). Conversely, those patients who sustained a head trauma with a GCS $\geq 13$, had a mortality rate of $1.2 \%$ (ISS of 10.2; well below major trauma levels). Expanded analysis of the data in Table 2 indicates only $4 \%$ of the patients with a $60 \%$ mortality risk had full priority 1 TTA, indicating the need for age based criteria in CHH's current TTA protocols (Table 3). Lack of age as a criterion for TTA increases the susceptibility geriatric trauma patients will be under-triaged and as our data shows, this drastically increases risk of mortality. ${ }^{1,13}$

As CHH's under-triage rates are above the acceptable standards set by ACS-COT, we recommend that an aged based criteria be added to our TTA protocol at our community based level II trauma center: priority 1 TTA for all patients 65 years or older sustaining head trauma with a GCS $\leq 12$ or suspicion of intracranial hemorrhage. It is the belief of the authors that this age specific criterion will improve emergency response to these potentially life-threatening injuries and may significantly improve the quality of care delivered to this vulnerable patient population. We will monitor the effect of a criteria change with additional projects with interim and long-term data analysis planned. 


\section{References}

1. Werner CA. The Old Population: 2010. In: Bureau USC, ed2011.

2. Spaniolas K, Cheng JD, Gestring ML, Sangosanya A, Stassen NA, Bankey PE. Ground level falls are associated with significant mortality in elderly patients. The Journal of trauma. 2010;69(4):821-825.

3. Goodmanson NW, Rosengart MR, Barnato AE, Sperry JL, Peitzman AB, Marshall GT. Defining geriatric trauma: when does age make a difference? Surgery. 2012;152(4):668-674; discussion 674-665.

4. Werman HA, Erskine T, Caterino J, Riebe JF, Valasek T. Development of statewide geriatric patients trauma triage criteria. Prehospital and disaster medicine. 2011;26(3):170-179.

5. Ichwan B, Darbha S, Shah MN, et al. Geriatric-specific triage criteria are more sensitive than standard adult criteria in identifying need for trauma center care in injured older adults. Annals of emergency medicine. 2015;65(1):92-100.e103.

6. Bonne S, Schuerer DJ. Trauma in the older adult: epidemiology and evolving geriatric trauma principles. Clinics in geriatric medicine. 2013;29(1):137-150.

7. Maxwell CA. Trauma in the geriatric population. Critical care nursing clinics of North America. 2015;27(2):183-197.

8. Demetriades D, Sava J, Alo K, et al. Old age as a criterion for trauma team activation. The Journal of trauma. 2001;51(4):754-756; discussion 756-757.

9. Demetriades D, Karaiskakis M, Velmahos G, et al. Effect on outcome of early intensive management of geriatric trauma patients. The British journal of surgery. 2002;89(10):1319-1322.

10. Lehmann R, Beekley A, Casey L, Salim A, Martin M. The impact of advanced age on trauma triage decisions and outcomes: a statewide analysis. American journal of surgery. 2009;197(5):571-574; discussion 574-575.

11. Rogers A, Rogers F, Bradburn E, et al. Old and undertriaged: a lethal combination. The American surgeon. 2012;78(6):711-715.

12. Calland JF, Ingraham AM, Martin N, et al. Evaluation and management of geriatric trauma: an Eastern Association for the Surgery of Trauma practice management guideline. The journal of trauma and acute care surgery. 2012;73(5 Suppl 4):S345-350.

13. Taylor MD, Tracy JK, Meyer W, Pasquale M, Napolitano LM. Trauma in the elderly: intensive care unit resource use and outcome. The Journal of trauma. 2002;53(3):407-414.

14. Maxwell CA, Mion LC, Mukherjee K, et al. Preinjury physical frailty and cognitive impairment among geriatric trauma patients determine postinjury functional recovery and survival. The journal of trauma and acute care surgery. 2016;80(2):195-203.

15. St John AE, Rowhani-Rahbar A, Arbabi S, Bulger EM. Role of trauma team activation in poor outcomes of elderly patients. The Journal of surgical research. 2016;203(1):95-102.

16. Mena JH, Sanchez AI, Rubiano AM, et al. Effect of the modified Glasgow Coma Scale score criteria for mild traumatic brain injury on mortality prediction: comparing classic and modified Glasgow Coma Scale score model scores of 13. The Journal of trauma. 2011;71(5):1185-1192; discussion 1193.

17. Stevenson M, Segui-Gomez M, Lescohier I, Di Scala C, McDonald-Smith G. An overview of the injury severity score and the new injury severity score. Injury prevention : journal of the International Society for Child and Adolescent Injury Prevention. 2001;7(1):10-13.

18. Samin OA, Civil ID. The New Injury Severity Score Versus the Injury Severity Score in Predicting Patient Outcome: A Comparative Evaluation on Trauma Service Patients of the Auckland Hospital. Annu Proc Assoc Adv Automot Med. 1999;43:1-15.

19. Surgeons ACo. ACS Committee on Trauma, Resources for the optimal care of the injured patient. 2014.

20. Nance M. National Trauma Data Bank 2011 Annual report. 2011.

21. Vincent G VV. The Next Four Decades the Older Population i nthe United States: 2010 to 2050. In: U.S. Dept. of Commerce EaSA, U.S. Censur Bureau, ed2010:25-1138.

22. Hildebrand F, Pape HC, Horst K, et al. Impact of age on the clinical outcomes of major trauma. European journal of trauma and emergency surgery : official publication of the European Trauma Society. 2016;42(3):317-332.

23. Escobar MA, Jr., Morris CJ. Using a multidisciplinary and evidence-based approach to decrease undertriage and overtriage of pediatric trauma patients. Journal of pediatric surgery. 2016.

24. Sammy I, Lecky F, Sutton A, Leaviss J, O'Cathain A. Factors affecting mortality in older trauma patients-A systematic review and meta-analysis. Injury. 2016;47(6):1170-1183. 
25. Clegg A, Young J, Iliffe S, Rikkert MO, Rockwood K. Frailty in elderly people. Lancet. 2013;381(9868):752-762.

26. Chehade M, Gill TK, Visvanathan R. Low Energy Trauma in Older Persons: Where to Next? The open orthopaedics journal. 2015;9:361-366.

27. Bhattacharya B, Maung A, Schuster K, Davis KA. The older they are the harder they fall: Injury patterns and outcomes by age after ground level falls. Injury. 2016.

28. Bradburn E, Rogers FB, Krasne M, et al. High-risk geriatric protocol: improving mortality in the elderly. The journal of trauma and acute care surgery. 2012;73(2):435-440.

29. Oreskovich MR, Howard JD, Copass MK, Carrico CJ. Geriatric trauma: injury patterns and outcome. The Journal of trauma. 1984;24(7):565-572.

30. Parker S, Afsharpad A. Ground-Level Geriatric Falls: A Not-So-Minor Mechanism of Injury. Case Reports in Orthopedics. 2014;2014.

31. Reddy S, Sharma R, Grotts J, Ferrigno L, Kaminski S. Incidence of intracranial hemorrhage and outcomes after ground-level falls in geriatric trauma patients taking preinjury anticoagulants and antiplatelet agents. The American surgeon. 2014;80(10):975-978.

32. Cheng PL, Lin HY, Lee YK, Hsu CY, Lee CC, Su YC. Higher mortality rates among the elderly with mild traumatic brain injury: a nationwide cohort study. Scandinavian journal of trauma, resuscitation and emergency medicine. 2014;22:7.

33. Hukkelhoven CW, Steyerberg EW, Rampen AJ, et al. Patient age and outcome following severe traumatic brain injury: an analysis of 5600 patients. Journal of neurosurgery. 2003;99(4):666-673.

34. Selassie AW, Cao Y, Church EC, Saunders LL, Krause J. Accelerated death rate in population-based cohort of persons with traumatic brain injury. The Journal of head trauma rehabilitation. 2014;29(3):E8e19.

35. Brooks JC, Strauss DJ, Shavelle RM, Paculdo DR, Hammond FM, Harrison-Felix CL. Long-term disability and survival in traumatic brain injury: results from the National Institute on Disability and Rehabilitation Research Model Systems. Archives of physical medicine and rehabilitation. 2013;94(11):2203-2209.

36. McIntyre A, Mehta S, Aubut J, Dijkers M, Teasell RW. Mortality among older adults after a traumatic brain injury: a meta-analysis. Brain injury. 2013;27(1):31-40. 